\title{
Analysis of urinary8-oxo-7,8-dihydro-purine-2'-deoxyribonucleosides
}

\section{by LC-MS/MS and improved ELISA}

Mark D. Evans ${ }^{1}$, Rajinder Singh ${ }^{2}$, Vilas Mistry ${ }^{1}$, Karendeep Sandhu $^{1}$, Peter B. Farmer ${ }^{2}$ and Marcus S. Cooke $e^{1,3}$.

${ }^{1}$ Radiation and Oxidative Stress Section, ${ }^{2}$ Cancer Biomarkers and Prevention Group, Dept. Cancer Studies and Molecular Medicine, University of Leicester, Leicester, UK; ${ }^{3} \&$ Dept. Genetics, University of Leicester, Leicester, UK.

\section{Author for Correspondence:}

Dr. M.D. Evans, Radiation \& Oxidative Stress Group, Dept. Cancer Studies \& Molecular Medicine, University of Leicester, RKCSB, Leicester Royal Infirmary, University Hospitals of Leicester NHS Trust, Leicester LE2 7LX. United Kingdom. Phone/Fax: +44 (0)116 252 5832. E-mail: mde2@le.ac.uk 


\begin{abstract}
Non-invasive monitoring of oxidative stress is highly desirable. Urinary 7,8-dihydro8-oxo-2'-deoxyguanosine (8-oxodG) is a biologically relevant and convenient analytical target. However, immunoassays can over-estimate levels of urinary 8oxodG. Measurement of more than one DNA oxidation product in urine would be advantageous in terms of mechanistic information. Urines samples were analysed for 8-oxodG by solid-phase extraction/LC-MS/MS and ELISA. The solid-phase extraction/LC-MS/MS assay was also applied to the analysis of urinary 7,8-dihydro-8oxo-2'-deoxyadenosine (8-oxodA). Concurring with previous reports, urinary 8oxodG measured by ELISA was significantly higher than levels measured by LCMS/MS. However, apparent improvement in the specificity of the commercially available Japanese Institute for the Control of Ageing (JaICA) ELISA brought mean LC-MS/MS and ELISA measurements of urinary 8-oxodG into agreement. Urinary 8-oxodA was undetectable in all urines, despite efficient recovery by solid phase extraction. Exploitation of the advantages of ELISA may be enhanced by a simple modification to the assay procedure, although chromatographic techniques still remain the 'gold standard' techniques for analysis of urinary 8-oxodG. Urinary 8-oxodA is either not present or below the limit of detection of our instrumentation.
\end{abstract}

Keywords: DNA oxidation, human urine, biomarkers, oxidised purines, immunoassay, mass spectrometry. 


\section{Introduction}

There is growing literature on the role of oxidative stress in a wide variety of malignant and non-malignant conditions $[1,2]$. Whilst many methods of analysis assess cellular markers of oxidative stress [3], these require an invasive procedure, limiting their use in very young, or very old, subjects and also impeding their use in large scale studies, where easy accessibility to samples and subsequent high throughput analysis are required. Long term storage of samples, such as those in biobanks, are likely to lead to the formation of adventitious damage, further limiting their use. These drawbacks may be avoided by using urinary markers of oxidative stress, and the analysis of 8-oxo-7,8-dihydro-2'-deoxyguanosine (8-oxodG), in particular has received widespread use, appearing eminently stable during long term storage [4]. The relatively large number of reports measuring urinary 8-oxodG as a biomarker of oxidative stress or even DNA damage, have been made in many cases without due consideration of the source of this lesion in urine. One can envisage three potential sources of nucleic acid oxidation products in urine: diet, cell death and repair (either of DNA or nucleotide pools) [5]. While the contributions of diet and cell death to the presence of urinary 8-oxoGua and 8-oxodG, for example, continue to be assessed, the prevailing view is that neither of these processes contribute significantly to the presence of these compounds in urine, but a full understanding of their origins is critically important to evaluating their utility as biomarkers. The examination of the origins of other lesions remains largely un-investigated, however.

Broadly speaking the approaches to urinary 8-oxodG determination are either chromatographic, such as liquid chromatography with tandem mass spectrometry [612], and high performance liquid chromatography with electrochemical detection [13- 
17]; or immunoassay, such as the commercial ELISA kit available from the Japanese Institute for the Control of Aging (JaICA) $[18,19]$. Immunoassay is clearly the most amenable approach, for most laboratories, not requiring expensive equipment, isotopically-labelled standards or specialist expertise. Furthermore, the ELISA from JaICA appears to be applicable to a variety of matrices: urine, serum, plasma, cell culture medium, saliva and DNA hydrolysates [19-22].

Although the JaICA ELISA is apparently applicable to several matrices in addition to urine, its use in saliva has recently been brought into question [19]. Furthermore, discrepancies in the levels of 8-oxodG in urine when measured by ELISA, compared to chromatographic techniques, has also questioned the utility of this ELISA (Figure 1). Early papers using the JaICA ELISA reported typical mean values of urinary 8oxodG ranging from 18.6 - $24.3 \mu \mathrm{g} / \mathrm{g}$ creatinine, whereas chromatographic techniques give mean levels of $0.79-2.13 \mu \mathrm{g} / \mathrm{g}$ creatinine in healthy subjects [23, 24]. More recently, a version of the ELISA, described as being 'improved', was released and typical values obtained with this ELISA were lower, ranging from $8.16-11.13 \mu \mathrm{g} / \mathrm{g}$ creatinine (Figure 1) [18, 25]. However, with strict temperature control advocated by Yoshida et al., we achieved levels of $4.12 \mu \mathrm{g} / \mathrm{g}$ creatinine, albeit still 4 times greater than LC-MS/MS analysis $(1.03 \mu \mathrm{g} / \mathrm{g}$ creatinine $)$ of the same samples $[18,19]$. Despite these differences in absolute levels, chromatographic and immunoassay approaches have been shown to correlate significantly [25-27], although with some notable exceptions $[19,28]$. Recent data from our laboratory have led us to conclude that whilst the ELISA is useful, the magnitude of the discrepancy in levels by the two approaches, suggests that the ELISA is, at present, unable to specifically determine 
absolute levels of urinary 8-oxodG [19]. As a consequence, we undertook an investigation of means by which the specificity of the ELISA might be enhanced.

The oxidation of DNA yields multiple base damage products, of which 8-oxodG is only one. It would therefore be valuable to measure more than one lesion in urine, not least as different DNA lesions have different cellular consequences [29]. Indeed, there are reports of the analysis of several oxidised base and 2'-deoxyribonucleoside products in urine [30]. The 2'-deoxyribonucleosides are preferred analytical targets in urine, largely for the same reasons that have often been cited with regard to the analysis of 8-oxodG versus the base, 8-oxo-7,8-dihydroguanine (8-oxoGua), i.e. the perceived dietary contribution to the latter, even if their provenance of the 2'deoxyribonucleosides is not as well defined. To further extend the repertoire of 2'deoxynucleoside oxidation products we have applied our recently reported solid-phase extraction (SPE) LC-MS/MS procedure to the analysis of urinary 8-oxo-7,8-dihydro2'-deoxyadenosine (8-oxodA) in a group of healthy subjects.

\section{Materials and methods}

Samples of human urine.

For the analysis of urinary 8-oxodG by ELISA and LC-MS/MS, spot urine samples were collected from a total of 20 healthy individuals (11 males and 9 females, mean age $=27$, age range $22-37$, following informed consent. For analysis of urinary 8-oxodA by LC-MS/MS, the above set of samples, plus an additional 10 samples, independently collected from healthy individuals were used, again following informed consent (16 males, 14 females, mean age $=29$, age range 20-52). All urine samples were stored at $-20^{\circ} \mathrm{C}$ until analysis. In order to provide a correction factor 
for urine concentration, aliquots of urine supernatant were also assayed for creatinine using the Jaffe method on an Olympus Olympus AU400 autoanalyser (Department of Chemical Pathology, Leicester Royal Infirmary, University Hospitals of Leicester NHS Trust, Leicester, UK), and urinary oxidised purine measurements corrected accordingly.

Solid-phase extraction (SPE) of 8-oxodG from urine and LC-MS/MS analysis.

The method for extraction of urinary 8 -oxodG, as well as the synthesis of $\left[{ }^{15} \mathrm{~N}_{5}\right] 8$ oxodG internal standard, has been described in detail elsewhere [19]. All urine samples were spiked with 24 pmol $\left[{ }^{15} \mathrm{~N}_{5}\right] 8$-oxodG prior to manipulation. We had previously processed differing volumes of urine, based upon creatinine content [23], but noted that creatinine is not a reliable indicator of 8-oxodG content [19], and so routinely processed $1.2 \mathrm{~mL}$ aliquots of all urine samples.

Subsequent treatment of the urine samples was exactly as reported previously [19]. Briefly, following purification by SPE ( $3 \mathrm{~mL}, 60 \mathrm{mg}$, Waters Oasis HLB, Waters Ltd, Elstree, UK), extracts were analysed by LC-MS/MS. The LC-MS/MS system consisted of a Waters Alliance 2695 separations module connected to a Micromass Quattro Ultima Platinum (Waters-Micromass Ltd., Manchester, UK) tandem quadrupole mass spectrometer with an electrospray interface. Selected reaction monitoring analysis was performed for the $[\mathrm{M}+\mathrm{H}]^{+}$ion to oxidised base $\left[\mathrm{B}+\mathrm{H}_{2}\right]^{+}$ transitions of 8-oxodG (m/z 284 to 168$)$ and $\left[{ }^{15} \mathrm{~N}_{5}\right] 8$-oxodG (m/z 289 to 173$)$. The level of 8-oxodG in each urine sample was determined from the ratio of the peak area of 8-oxodG to that of the internal standard. 
ELISA of urinary 8-oxodG.

Following thawing and centrifugation (300g, 10 mins), urine sample supernatants were applied to the competitive ELISA plate (50 $\mu \mathrm{L} /$ well) according to the protocol supplied by JaICA (Fukuroi, Japan). No other pre-treatment of the samples was performed. In line with the recommendation of Yoshida et al., we again applied strict temperature control to the antibody incubations [18]. Additionally, we investigated the effect of incubation temperature upon urinary 8-oxodG results, performing the primary antibody incubation step at $4{ }^{\circ} \mathrm{C}$ overnight; or $37^{\circ} \mathrm{C}$ for one hour, the latter as recommended by JaICA.

\section{Further characterisation of 8-oxodG ELISA primary antibody}

Stock solutions of 8-oxodG, 8-chloroguanosine (8-chloroGuo; Axxora Platform, Nottingham, UK), 8-oxoguanosine (8-oxoGuo; Calbiochem, Nottingham, UK) and 8oxo-7,8-dihydro-2'-deoxyguanosine-5'-monophosphate (8-oxodGMP) were made up to a concentration of $5 \mathrm{mM}$ in ultrapure water. These stocks were then diluted to a final concentration of $20 \mu \mathrm{M}$ with urine from the same, single urine sample. These standards were further serially diluted, again in the same urine, across the ELISA plate, to a range of final concentrations $(20-0.000256 \mu \mathrm{M})$. Antibody recognition of a single-stranded oligodeoxyribonucleotide containing a single 8-oxodG was also investigated. The oligomer (22-mer, 5' GAACTAGTOATCCCCGGGCTGC 3', where $\mathbf{O}$ is 8-oxodG; (Trevigen, Gaithersburg, USA) was made up to a maximal concentration of $243.9 \mathrm{nM}$, which resulted in a final concentration of $9.4 \mathrm{nM} \mathrm{8-}$ oxodG, similar to that reported in a previous study [31], prior to serial dilution across the ELISA plate to a range of final concentrations $\left(9.4-1.2 \times 10^{-7} \mathrm{nM}\right)$. The ELISA 
was then performed as described above, and the effect of primary antibody (N45.1) incubation at $37^{\circ} \mathrm{C}$ and $4{ }^{\circ} \mathrm{C}$, also investigated.

\section{Preparation of $\left[{ }^{15} N_{5}\right] 8-$ oxodA}

Synthesis of $\left[{ }^{15} \mathrm{~N}_{5}\right] 8$-oxodA was performed using $600 \mathrm{~Gy}$ of $\gamma$-irradiation, delivered by a ${ }^{60}$ Co-source at the University of Leicester, to a $1 \mathrm{mg} / \mathrm{ml}$ solution of $\left[{ }^{15} \mathrm{~N}_{5}\right] 2$ 'deoxyadenosine ( $>98 \%{ }^{15} \mathrm{~N}$; Spectra Stable Isotopes, Columbia, MD, USA) in water saturated with nitrous oxide. An aliquot of irradiated material was analysed by HPLC, and the identity of 8-oxodA confirmed based upon comparison of the retention time and spectral properties to that of an authentic, commercially-obtained standard. Isotopically-labelled 8-oxodA was isolated on a Columbus, $5 \mu \mathrm{m}_{8}$ semi-preparative column, $250 \times 10 \mathrm{~mm}$ (Phenomenex, Macclesfield, UK), mobile phase was $10 \%$ methanol in water, flow rate $5 \mathrm{~mL} / \mathrm{min}$. Collected material was freeze-dried, reconstituted in water and the concentration determined via absorbance at $270 \mathrm{~nm}\left(\varepsilon_{270}\right.$ $\left.=12764 \mathrm{M}^{-1} \mathrm{~cm}^{-1}\right),\left[{ }^{15} \mathrm{~N}_{5}\right] 8$-oxodA was also quantified by LC-MS/MS by comparison to a known amount of unlabelled commercially obtained standard, which additionally verified the identity of labelled material (unlabelled material was present at $\sim 1.0 \%$, undetectable at the levels used for internal standardisation of samples). Aliquots of standard in solution were stored at $-80{ }^{\circ} \mathrm{C}$.

\section{Solid-phase extraction of urinary 8-oxodA.}

The SPE procedure used was identical to that used for urinary 8-oxodG, with $1.2 \mathrm{~mL}$ of urine processed following addition of $12 \mathrm{pmol}\left[{ }^{15} \mathrm{~N}_{5}\right] 8$-oxodA to all samples prior to manipulation. The recovery of 8-oxodA from urine by SPE was assessed by spiking $1.2 \mathrm{~mL}$ urine samples with additional unlabelled 8-oxodA $(0,50,100,200$ 
pmol), performing SPE and then adding $12 \mathrm{pmol}\left[{ }^{15} \mathrm{~N}_{5}\right] 8$-oxodA post-SPE. A second set of urine samples were spiked with labelled and unlabelled 8-oxodA after SPE. Recovery was calculated according to:

(Peak area, unlabelled 8-oxodA added before SPE/Peak area $\left[{ }^{15} \mathrm{~N}_{5}\right]$-oxodA) $\times 100$ (Peak area, unlabelled 8-oxodA added after SPE/Peak area $\left[{ }^{15} \mathrm{~N}_{5}\right] 8$-oxodA)

The recovery experiments were done using urine samples from 3 individuals. The limit of detection was determined by reconstituting urine extracts (5 different urine samples) in aqueous solutions containing various concentrations of $\left[{ }^{15} \mathrm{~N}_{5}\right]-8$ oxodA prior to LC-MS/MS analysis. A signal:noise ratio of $\geq 3$ was used as a cut-off for the determination of the limit of detection. We have previously reported LOD, recovery and linearity of response data for 8-oxodG using this method [19].

\section{LC-MS/MS analysis of urinary 8-oxodA}

The same instrument as used for urinary 8-oxodG analysis was used for the analysis of urinary 8-oxodA. The electrospray source was maintained at $110{ }^{\circ} \mathrm{C}$ and the desolvation temperature at $350{ }^{\circ} \mathrm{C}$. Nitrogen was used as the desolvation gas (650 $\mathrm{L} / \mathrm{h}$ ), with the cone gas was set to zero. The capillary voltage was set at $3.20 \mathrm{kV}$. The cone and RF1 lens voltages were $42 \mathrm{~V}$ and $30 \mathrm{~V}$, respectively. The mass spectrometer was tuned by using an 8 -oxodA $(10 \mathrm{pmol} / \mu \mathrm{L})$ standard solution dissolved in $0.1 \%$ acetic acid/methanol $(85: 15, \mathrm{v} / \mathrm{v})$ introduced by continuous infusion at a flow rate of $10 \mu \mathrm{L} / \mathrm{min}$ with a Harvard model 22 syringe pump (Havard Apparatus Ltd., Edenbridge, UK). A $10 \mu \mathrm{L}$ aliquot of the purified sample was injected onto a Synergi Fusion-RP 80A C $18(4 \mu, 250 \times 2.0 \mathrm{~mm})$ column (Phenomenex, Macclesfield, UK) and a Synergi Fusion-RP $80 \mathrm{C}_{18}(4 \mu, 4.0 \times 2.0 \mathrm{~mm})$ guard column attached to a 
KrudKatcher disposable pre-column $(0.5 \mu \mathrm{m})$ filter. The column was eluted isocratically with $0.1 \%$ acetic acid/methanol $(85: 15, \mathrm{v} / \mathrm{v})$ at a flow rate of $120 \mu \mathrm{L} / \mathrm{min}$. The collision gas was argon (indicated cell pressure $2.0 \times 10^{-3} \mathrm{mbar}$ ) and the collision energy set at $12 \mathrm{eV}$. The dwell time was set to $200 \mathrm{~ms}$ and the resolution was two $\mathrm{m} / \mathrm{z}$ units at peak base. Selected reaction monitoring (SRM) analysis was performed for the $[\mathrm{M}+\mathrm{H}]^{+}$ion to oxidised base $\left[\mathrm{B}+\mathrm{H}_{2}\right]^{+}$transitions of 8-oxodA $(\mathrm{m} / \mathrm{z} 268$ to 152$)$ and the stable isotope internal standard $\left[{ }^{15} \mathrm{~N}_{5}\right] 8-\operatorname{oxodA}(\mathrm{m} / \mathrm{z} 273$ to 157$)$.

Statistical analysis.

Graphs and statistical analyses were performed by GraphPad Prism v.4.03 (GraphPad Software, San Diego, USA). The analysis of differences between datasets were made using the Mann-Whitney U-test and data correlations tested using Spearman's rank correlation.

\section{Results}

\section{ELISA and LC-MS/MS of urinary 8-oxodG}

We have attempted to enhance the specificity of N45.1 binding in the JaICA competitive ELISA, by performing the primary antibody incubation step at $4{ }^{\circ} \mathrm{C}$, overnight, rather than for one hour at $37{ }^{\circ} \mathrm{C}$ (Figure 2). Apart from at low concentrations of 8-oxodG $(<2 \mathrm{ng} / \mathrm{mL})$, altering the incubation temperature produced only subtle differences between the calibration curves (Figure 2). Mean urinary 8oxodG values were significantly lower following ELISA primary antibody incubation at $4{ }^{\circ} \mathrm{C}$ compared to $37{ }^{\circ} \mathrm{C}(3.44 \pm 1.62 \mathrm{pmol} / \mu \mathrm{mol}$ creatinine and $7.86 \pm 3.92$ $\mathrm{pmol} / \mu \mathrm{mol}$ creatinine, respectively, $\mathrm{p}<0.0001$; Figure 3 ). While the mean level of urinary 8-oxodG was significantly $(\mathrm{p}<0.0024)$ lower when measured by LC-MS/MS 
$\left(4.65 \pm 2.09 \mathrm{pmol} / \mu \mathrm{mol}\right.$ creatinine) compared to ELISA at $37{ }^{\circ} \mathrm{C}$, this was not statistically different from the mean level of urinary 8-oxodG assessed by ELISA at 4 ${ }^{\circ} \mathrm{C}(\mathrm{p}=0.054)$. Linear regression analysis revealed a good correlation between 8oxodG values obtained at $37{ }^{\circ} \mathrm{C}$ and $4{ }^{\circ} \mathrm{C}\left(\mathrm{r}_{\mathrm{s}}=0.73 ; \mathrm{p}<0.0009\right.$; Figure 4A). Linear regression analysis also revealed a significant correlation between $4{ }^{\circ} \mathrm{C}$ ELISA 8oxodG values, and those obtained by LC-MS/MS $\left(r_{s}=0.65 ; p<0.005\right.$; Figure 4B).

\section{Further characterisation of 8-oxodG ELISA primary antibody}

Competitive ELISA was used to evaluate the ability of putative antigens to compete with 8-oxodG, bound to the ELISA plate, for primary antibody binding. There was no recognition of 8-chloroGuo, following primary antibody incubation at $37{ }^{\circ} \mathrm{C}$ or $4{ }^{\circ} \mathrm{C}$, even at the highest concentration of competitor ( $20 \mu \mathrm{M}$; Figures $5 \mathrm{~A}$ and $5 \mathrm{~B})$. It was only at the highest concentration of 8-oxodG-containing oligomer (9.4 $\mu \mathrm{M}$ 8-oxodG), and at $37^{\circ} \mathrm{C}$, that some inhibition of $\mathrm{N} 45.1$ was noted (Figure 5A). In contrast 8oxoGuo appeared to compete effectively over most of the concentrations used, with an $\mathrm{IC}_{50-}$ (concentration of competitor at which $50 \%$ of antibody binding is inhibited) of $0.867 \mu \mathrm{M}$. As expected, 8 -oxodG proved to be a highly effective competitor, with an $\mathrm{IC}_{50}$ of $0.078 \mu \mathrm{M}$, approximately one tenth that of 8-oxoGuo. Surprisingly, 8oxodGMP was over two-and-a-half times a more effective a competitor than 8-oxdG at $37^{\circ} \mathrm{C}$, with an $\mathrm{IC}_{50}$ of 0.029 (Figure $5 \mathrm{~A}$ ).

The $\mathrm{IC}_{50}$ values for 8-oxoGuo, 8-oxodG and 8-oxodGMP were all much lower at 4 ${ }^{\circ} \mathrm{C}$, compared to $37{ }^{\circ} \mathrm{C}(0.022,0.0009$ and $0.0007 \mu \mathrm{M}$, respectively), indicating greater inhibition at much lower concentrations of competitor (Figure 5B). The 
results at $4{ }^{\circ} \mathrm{C}$ also revealed that, in contrast to at $37^{\circ} \mathrm{C}$, there was no significant difference in $\mathrm{IC}_{50}$ between 8-oxodG and 8-oxodGMP, at any concentration. Furthermore, the $\mathrm{IC}_{50}$ values for these two compounds were approximately 25 times less than 8-oxoGuo.

Analysis of urinary 8-oxodA by SPE, LC-MS/MS

We were unable to detect 8-oxodA in any of the urine samples examined (example chromatogram in Figure 6), this was despite efficient recovery of 8-oxodA from urine by the SPE procedure $(95 \pm 10 \%)$. Our method was also unable to detect 8 -oxodA in urine by increasing the amount of urine processed to $3.2 \mathrm{~mL}$ (for two randomly selected healthy subjects) or performing a double SPE, using $3.2 \mathrm{~mL}$ urine, i.e. processing collected material from one SPE extraction through a second SPE column. With a limit of detection for 8 -oxodA by our method of $10 \mathrm{fmol}$ on column, this implies that 8 -oxodA is present in urine at less than $20 \mathrm{fmol} / \mathrm{mL}$ urine.

\section{Discussion}

The development of the JaICA competitive ELISA for measurement of 8-oxodG in extracellular fluids has lead to widespread use in a variety of pathologies and extracellular fluids. Such an assay format has distinct advantages in terms of equipment requirements, user training and sample-throughput, compared to chromatographic techniques, such as HPLC-electrochemical detection or LC-mass spectrometry. However, for many years the ELISA has consistently given higher values for urinary 8-oxodG compared to chromatographic procedures and, from the viewpoint of our laboratory, this has partly been interpreted as recognition by the primary antibody (designated N45.1) of 8-oxodG in forms other than the free 2'- 
deoxyribonucleoside, e.g. 8-oxodG-containing oligodeoxyribonucleotides, or 8oxodGMP. Previously unpublished data from our laboratory indicated that N45.1 can recognise single-stranded, 8-oxodG-containing oligomers, which is supported by our $37^{\circ} \mathrm{C}$ findings reported here. It appears that $\mathrm{N} 45.1$ is specific for 8-oxodG, and of the compounds tested previously as potential competitors, the closest are 8-oxo-guanosine and 8-mercapto-2'-deoxyguanosine [32]. The latter compound has limited biological relevance and the former needs to be present at $\sim 10$ times the concentration of 8 oxodG, at $37^{\circ} \mathrm{C}$, to be an effective competitor, in close agreement with our findings reported here. Results for the ELISA at $4{ }^{\circ} \mathrm{C}$ would suggest that 8-oxoGuo needs to be present at 25 times the concentration of 8-oxodG, to affect equal competition, in both cases a situation, seemingly, not realised in urine [31]. This is the first report to demonstrate that 8-oxodGMP is an effective competitor for N45.1 binding, being over two-and-a-half times more effective than 8-oxodG, when the ELISA is performed at $37^{\circ} \mathrm{C}$. We propose that the basis for this result might derive from the immunogen used in the production of N45.1. Conjugation of 8-oxodG, via a succinamide linkage, to a carrier protein for immunisation would generate a structure with some similarities to 8-oxodGMP. This is an interesting observation, and adds to the criteria that determine which antigens are recognised by $\mathrm{N} 45.1$. At $4{ }^{\circ} \mathrm{C}$, however, this discrimination is lost, suggesting that it is a relatively weak interaction which accounts for the, albeit appreciable, selectivity for 8-oxodGMP over 8-oxodG. Previously, reactivity of 8-bromoGuo has been investigated, and shown not to compete [32], likewise we show here that 8-chloroGuo does not compete for antibody binding, irrespective of incubation temperature. 
It could be possible, that because of the competitive format of the 8-oxodG ELISA, species structurally dissimilar to 8-oxodG may interfere with N45.1 binding yielding false-positive signals. Such species could include proteins and carbohydrates. In fact, while this ELISA is recommended for the analysis of 8-oxodG in protein rich matrices such as serum, JaICA released further advice for the use of their kit in this situation, requiring sample clean-up using $10 \mathrm{kDa}$ cut-off filter membranes, prior to use in the ELISA. Our speculation that the presence of relatively high levels of protein and carbohydrate could interfere with this assay was reinforced recently by our studies on salivary 8-oxodG [19]. Antibodies have also found use in immnoaffinity clean-up of urine samples prior to chromatographic analysis. However, there would seem to be significant advantages to using SPE, which avoids the need for ready availability of relatively large quantities of appropriately characterised, specific and potentially expensive antibodies, which are more liable to suffer from interference, particularly when analysing low molecular weight species in complex biological matrices.

Overnight incubation of N45.1 with pure standards at low temperature does not appear to markedly affect its binding to 8-oxodG (Fig. 2), i.e. the primary, and strongest, molecular interactions between antibody and antigen. Longer incubation, at lower temperatures, perhaps minimise secondary, weaker, and more easily reversible antibody-antigen interactions, by allowing the system greater time to reach equilibrium and it would also be expected that the stronger interactions would be those that would also form more easily at lower temperatures. This longer, low temperature, incubation period increases the specificity of the antibody-antigen interactions and brings the ELISA data into significant agreement with the chromatographic data for our samples (Fig. 3). Whilst these data demonstrate that 
8-oxodGMP and 8-oxoGuo may significantly compete, and that 8-oxodG-containing, single-stranded oligomers may contribute to weak interactions, with N45.1, the experimental evidence of the presence of these in urine, at concentrations sufficient to be detected by the ELISA, is presently minimal $[31,33]$. Furthermore, these weak interactions can be prevented by incubation of N45.1 with samples in the initial step of the ELISA at least overnight (ca. 15-18hrs), at 4-6 ${ }^{\circ} \mathrm{C}$. On this basis, for urine, at least, we would recommend that this step is adopted.

Clearly however, our data still reveal discrepancies between ELISA and LC-MS/MS determinations of individual urinary 8-oxodG values, as although the mean level of urinary 8-oxodG is not statistically different between the two methods, there is still not a perfect correlation between values. Thus there is a considerable element of individual variability in the urines that is difficult to control for in competitive ELISA. Until the issues with this, and perhaps other ELISA methods, are rectified, chromatographic methods, preferably with robust internal standardisation and compound identification techniques, have to remain as the 'gold standard' procedures for determination of urinary 8-oxodG, as there is no way of accurately ascribing differences between individuals to urinary 8-oxodG, specifically, by ELISA.

Our apparent inability to detect 8-oxodA in any of the 30 urine samples from healthy donors is consistent with the one previous report of an attempt to detect this lesion in urine [8]. We suspect that this is due to sensitivity limitations of current instrumentation, however the SPE LC-MS/MS methodology we report has all the attendant advantages of mass spectrometry in terms of internal standardisation, peak identification and we are confident of the ability of the SPE step to efficiently extract 
8-oxodA from urine with a limit of detection of comparable sensitivity to 8-oxodG. In contrast to the 2'-deoxyribonucleoside, the base, 8-oxo-7,8-dihydroadenine (8oxoAde), has been reported in urine, although it was present in the lowest amounts out of five base lesions examined [9]. However, the exact processes leading to the release of 8-oxoAde from DNA remain obscure. Our laboratory was amongst the first to consider the activity of the mutT homologue, MTH1, and perhaps other Nudix hydrolases, as a rational and significant source of extracellular 8-oxodG, following hydrolysis of 8-oxodGTP formed in nucleotide pools [22, 24, 34]. Thus, along with the biological importance of limiting the availability of modified and potentially mutagenic dNTPs, additional meaning may become ascribed to urinary 8-oxodG levels. The very low levels of 8-oxodA in urine, compared to 8-oxodG, perhaps reflect the higher oxidation potential of adenine. Even though 8-oxodATP is a substrate for MTH1, if formed to any extent in nucleotide pools, it is likely to be present at levels present even lower than 8-oxodGTP. There also remains the possibility that further oxidation of 8-oxodA could occur, as has been reported for some other oxidation-derived lesions, such as 8-oxodG, thus diminishing levels of the parent lesion even further [35].

Only with validated analytical tools will we be able to better understand the significance of these urinary markers of nucleic acid oxidation. In this paper we have shown that the specificity of a commercially available competitive ELISA for 8oxodG may be improved by a minor alteration to the protocol, to bring the data into better agreement with an analytically robust technique such as LC-MS/MS. However, there still remain issues regarding the applicability of these immunoassays to selected biological matrices, e.g. saliva and also determining the impacts of variation in 
individual urine samples on the ELISA. The potential exists to measure more than one nucleic acid oxidation product in a single chromatographic run, for example by LC-MS/MS with all its attendant advantages of internal standardisation and analyte identification. However, in agreement with one other report, urinary 8-oxodA appears undetectable with our instrumentation, either because it is not present or, the more likely scenario that it is below the limit of detection. 
Acknowledgements: The authors are partners of ECNIS (Environmental Cancer Risk, Nutrition and Individual Susceptibility), a network of excellence operating within the European Union $6^{\text {th }}$ Framework Program, Priority 5:"Food Quality and Safety" (Contract No 513943). Financial support from ECNIS is greatly appreciated. PBF and RS also acknowledge the UK Medical Research Council for financial support. 


\section{References}

[1] Cooke MS, Olinski R, Evans MD. Does measurement of oxidative damage to DNA have clinical significance? Clin Chim Acta 2006;365:30-49.

[2] Wu LL, Chiou CC, Chang PY, Wu JT. Urinary 8-OHdG: a marker of oxidative stress to DNA and a risk factor for cancer, atherosclerosis and diabetics. Clin Chim Acta 2004;339:1-9.

[3] Guetens G, De Boeck G, Highley M, van Oosterom AT, de Bruijn EA. Oxidative DNA damage: biological significance and methods of analysis. Crit Rev Clin Lab Sci 2002;39:331-457.

[4] Loft S, Svoboda P, Kasai H, Tjonneland A, Vogel U, Moller P, et al. Prospective study of 8-oxo-7,8-dihydro-2'-deoxyguanosine excretion and the risk of lung cancer. Carcinogenesis 2006;27:1245-50.

[5] Cooke MS, Olinski R, Loft S. Measurement and meaning of oxidatively modified DNA lesions in urine. Cancer Epidemiol Biomarkers Prev 2008;17:3-14.

[6] Hu CW, Wang CJ, Chang LW, Chao MR. Clinical-scale high-throughput analysis of urinary 8-oxo-7,8-dihydro-2'-deoxyguanosine by isotope-dilution liquid chromatography-tandem mass spectrometry with on-line solid-phase extraction. Clin Chem 2006;52:1381-8.

[7] Sabatini L, Barbieri A, Tosi M, Roda A, Violante FS. A method for routine quantitation of urinary 8-hydroxy-2'-deoxyguanosine based on solid-phase extraction and micro-high-performance liquid chromatography/electrospray ionization tandem mass spectrometry. Rapid Commun Mass Spectrom 2005;19:147-52.

[8] Weimann A, Belling D, Poulsen HE. Measurement of 8-oxo-2'deoxyguanosine and 8-oxo-2'-deoxyadenosine in DNA and human urine by high performance liquid chromatography-electrospray tandem mass spectrometry. Free Radic Biol Med 2001;30:757-64.

[9] Ravanat JL, Guicherd P, Tuce Z, Cadet J. Simultaneous determination of five oxidative DNA lesions in human urine. Chem Res Toxicol 1999;12:802-8.

[10] Crow B, Bishop M, Kovalcik K, Norton D, George J, Bralley JA. A simple and cost effective method for the quantification of 8-hydroxy-2'deoxyguanosine from urine using liquid chromatography tandem mass spectrometry. Biomed Chromatogr 2007.

[11] Malayappan B, Garrett TJ, Segal M, Leeuwenburgh C. Urinary analysis of 8oxoguanine, 8-oxoguanosine, fapy-guanine and 8-oxo-2'-deoxyguanosine by high-performance liquid chromatography-electrospray tandem mass spectrometry as a measure of oxidative stress. J Chromatogr A 2007;1167:5462 .

[12] Harri M, Kasai H, Mori T, Tornaeus J, Savela K, Peltonen K. Analysis of 8hydroxy-2'-deoxyguanosine in urine using high-performance liquid chromatography-electrospray tandem mass spectrometry. J Chromatogr B Analyt Technol Biomed Life Sci 2007;853:242-6.

[13] Kasai H. A new automated method to analyze urinary 8hydroxydeoxyguanosine by a high-performance liquid chromatographyelectrochemical detector system. J Radiat Res (Tokyo) 2003;44:185-9. 
[14] Shigenaga MK, Ames BN. Assays for 8-hydroxy-2'-deoxyguanosine: a biomarker of in vivo oxidative DNA damage. Free Radic Biol Med 1991;10:211-6.

[15] Germadnik D, Pilger A, Rudiger HW. Assay for the determination of urinary 8-hydroxy-2'-deoxyguanosine by high-performance liquid chromatography with electrochemical detection. J Chromatogr B Biomed Sci Appl 1997;689:399-403.

[16] De Martinis BS, de Lourdes Pires Bianchi M. Methodology for urinary 8hydroxy-2'-deoxyguanosine analysis by HPLC with electrochemical detection. Pharmacol Res 2002;46:129-31.

[17] Bogdanov MB, Beal MF, McCabe DR, Griffin RM, Matson WR. A carbon column-based liquid chromatography electrochemical approach to routine 8hydroxy-2'-deoxyguanosine measurements in urine and other biologic matrices: a one-year evaluation of methods. Free Radic Biol Med 1999;27:647-66.

[18] Yoshida R, Ogawa Y, Kasai H. Urinary 8-oxo-7,8-dihydro-2'-deoxyguanosine values measured by an ELISA correlated well with measurements by highperformance liquid chromatography with electrochemical detection. Cancer Epidemiol Biomarkers Prev 2002;11:1076-81.

[19] Cooke MS, Singh R, Hall GK, Mistry V, Duarte TL, Farmer PB, et al. Evaluation of enzyme-linked immunosorbent assay and liquid chromatography-tandem mass spectrometry methodology for the analysis of 8oxo-7,8-dihydro-2'-deoxyguanosine in saliva and urine. Free Radic Biol Med 2006;41:1829-36.

[20] Breton J, Sichel F, Bianchini F, Prevost V. Measurement of 8-hydroxy-2'deoxyguanosine by a commercially available ELISA test: comparison with HPLC/electrochemical detection in calf thymus DNA and determination in human serum Analytical Letters 2003;36:123-34.

[21] Kantha SS, Wada S, Tanaka H, Takeuchi M, Watabe S, Ochi H. Carnosine sustains the retention of cell morphology in continuous fibroblast culture subjected to nutritional insult. Biochem Biophys Res Commun 1996;223:27882.

[22] Haghdoost S, Czene S, Naslund I, Skog S, Harms-Ringdahl M. Extracellular 8-oxo-dG as a sensitive parameter for oxidative stress in vivo and in vitro. Free Radic Res 2005;39:153-62.

[23] Lin HS, Jenner AM, Ong CN, Huang SH, Whiteman M, Halliwell B. A highthroughput and sensitive methodology for the quantification of urinary 8hydroxy-2'-deoxyguanosine: measurement with gas chromatography-mass spectrometry after single solid-phase extraction. Biochem J 2004;380:541-8.

[24] Cooke MS, Evans MD, Herbert KE, Lunec J. Urinary 8-oxo-2'deoxyguanosine--source, significance and supplements. Free Radic Res 2000;32:381-97.

[25] Shimoi K, Kasai H, Yokota N, Toyokuni S, Kinae N. Comparison between high-performance liquid chromatography and enzyme-linked immunosorbent assay for the determination of 8-hydroxy-2'-deoxyguanosine in human urine. Cancer Epidemiol Biomarkers Prev 2002;11:767-70.

[26] $\mathrm{Hu} \mathrm{CW}$, Wu MT, Chao MR, Pan CH, Wang CJ, Swenberg JA, et al. Comparison of analyses of urinary 8-hydroxy-2'-deoxyguanosine by isotopedilution liquid chromatography with electrospray tandem mass spectrometry 
and by enzyme-linked immunosorbent assay. Rapid Commun Mass Spectrom 2004;18:505-10.

[27] Cooke MS, Rozalski R, Dove R, Gackowski D, Siomek A, Evans MD, et al. Evidence for attenuated cellular 8-oxo-7,8-dihydro-2'-deoxyguanosine removal in cancer patients. Biol Chem 2006;387:393-400.

[28] Prieme H, Loft S, Cutler RG, Poulsen HE. Measurement of oxidative DNA injury in humans: evaluation of a commercially available ELISA assay. Natural antioxidants and food quality in atheroslcerosis and cancer prevention 1996.

[29] Evans MD, Dizdaroglu M, Cooke MS. Oxidative DNA damage and disease: induction, repair and significance. Mutat Res 2004;567:1-61.

[30] Cooke MS, Lunec J, Evans MD. Progress in the analysis of urinary oxidative DNA damage. Free Radic Biol Med 2002;33:1601-14.

[31] Weimann A, Riis B, Poulsen HE. Oligonucleotides in human urine do not contain 8-oxo-7,8-dihydrodeoxyguanosine. Free Radic Biol Med 2004;36:1378-82.

[32] Toyokuni S, Tanaka T, Hattori Y, Nishiyama Y, Yoshida A, Uchida K, et al. Quantitative immunohistochemical determination of 8-hydroxy-2'deoxyguanosine by a monoclonal antibody N45.1: its application to ferric nitrilotriacetate-induced renal carcinogenesis model. Lab Invest 1997;76:36574.

[33] Patel PR, Bevan RJ, Mistry N, Lunec J. Evidence of oligonucleotides containing 8-hydroxy-2'-deoxyguanosine in human urine. Free Radic Biol Med 2007;42:552-8.

[34] Hayakawa H, Taketomi A, Sakumi K, Kuwano M, Sekiguchi M. Generation and elimination of 8-oxo-7,8-dihydro-2'-deoxyguanosine 5'-triphosphate, a mutagenic substrate for DNA synthesis, in human cells. Biochemistry 1995;34:89-95.

[35] Hah SS, Mundt JM, Kim HM, Sumbad RA, Turteltaub KW, Henderson PT. Measurement of 7,8-dihydro-8-oxo-2'-deoxyguanosine metabolism in MCF-7 cells at low concentrations using accelerator mass spectrometry. Proc Natl Acad Sci U S A 2007;104:11203-8.

[36] Saeki T, Ichiba M, Tanabe N, Ueki M, Okamoto K, Matsunaga Y, et al. Expression of oxidative stress-related molecules in circulating leukocytes and urine in patients with chronic viral hepatitis. Liver Int 2006;26:157-65.

[37] Hata I, Kaji M, Hirano S, Shigematsu Y, Tsukahara H, Mayumi M. Urinary oxidative stress markers in young patients with type 1 diabetes. Pediatr Int 2006;48:58-61.

[38] Lee KH, Bartsch H, Nair J, Yoo DH, Hong YC, Cho SH, et al. Effect of shortterm fasting on urinary excretion of primary lipid peroxidation products and on markers of oxidative DNA damage in healthy women. Carcinogenesis 2006;27:1398-403.

[39] Maeshima E, Liang XM, Otani H, Mune M, Yukawa S. Effect of environmental changes on oxidative deoxyribonucleic acid (DNA) damage in systemic lupus erythematosus. Arch Environ Health 2002;57:425-8.

[40] Liu AL, Lu WQ, Wang ZZ, Chen WH, Lu WH, Yuan J, et al. Elevated levels of urinary 8-hydroxy-2 -deoxyguanosine, lymphocytic micronuclei, and serum glutathione S-transferase in workers exposed to coke oven emissions. Environ Health Perspect 2006;114:673-7. 
[41] Wong RH, Kuo CY, Hsu ML, Wang TY, Chang PI, Wu TH, et al. Increased levels of 8-hydroxy-2 -deoxyguanosine attributable to carcinogenic metal exposure among schoolchildren. Environ Health Perspect 2005;113:1386-90.

[42] Drury JA, Jeffers G, Cooke RW. Urinary 8-hydroxydeoxyguanosine in infants and children. Free Radic Res 1998;28:423-8.

[43] Dhawan V, Jain S. Garlic supplementation prevents oxidative DNA damage in essential hypertension. Mol Cell Biochem 2005;275:85-94.

[44] Pilger A, Germadnik D, Riedel K, Meger-Kossien I, Scherer G, Rudiger HW. Longitudinal study of urinary 8-hydroxy-2'-deoxyguanosine excretion in healthy adults. Free Radic Res 2001;35:273-80.

[45] Kim M-H, Moon H-R, Hong S-Y. Determination of urinary 8-hydroxy-2'deoxyguanosine as a DNA damage marker. American Clinical Laboratory 2001:42-5.

[46] Bogdanov M, Brown RH, Matson W, Smart R, Hayden D, O'Donnell H, et al. Increased oxidative damage to DNA in ALS patients. Free Radic Biol Med 2000;29:652-8.

[47] Thompson HJ, Heimendinger J, Haegele A, Sedlacek SM, Gillette C, O'Neill $\mathrm{C}$, et al. Effect of increased vegetable and fruit consumption on markers of oxidative cellular damage. Carcinogenesis 1999;20:2261-6.

[48] Leinonen J, Lehtimaki T, Toyokuni S, Okada K, Tanaka T, Hiai H, et al. New biomarker evidence of oxidative DNA damage in patients with non-insulindependent diabetes mellitus. FEBS Lett 1997;417:150-2.

[49] Tsuboi H, Kouda K, Takeuchi H, Takigawa M, Masamoto Y, Takeuchi M, et al. 8-hydroxydeoxyguanosine in urine as an index of oxidative damage to DNA in the evaluation of atopic dermatitis. Br J Dermatol 1998;138:1033-5.

[50] Witherell HL, Hiatt RA, Replogle M, Parsonnet J. Helicobacter pylori infection and urinary excretion of 8-hydroxy-2-deoxyguanosine, an oxidative DNA adduct. Cancer Epidemiol Biomarkers Prev 1998;7:91-6.

[51] Miwa M, Matsumaru H, Akimoto Y, Naito S, Ochi H. Quantitative determination of urinary 8-hydroxy-2'-deoxyguanosine level in healthy Japanese volunteers. Biofactors 2004;22:249-53.

[52] Lai CH, Liou SH, Lin HC, Shih TS, Tsai PJ, Chen JS, et al. Exposure to traffic exhausts and oxidative DNA damage. Occup Environ Med 2005;62:216-22. 


\section{Figure legends}

Figure 1: Representation of mean 8-oxodG levels, in spot urines, determined by Chromatography techniques ('Chromatography'), (ii) the early version of the JaICA ELISA ('ELISA'), and (iii) the improved (post-2002) JaICA ELISA ('ELISA improved'). Results are expressed as a ratio between 8-oxodG and creatinine. The information contained in this figure is a composite of information derived from multiple literature references [4, 25, 36-52].

Figure 2: Calibration curves for the 8-oxodG ELISA when the primary antibody has been incubated for (i) one hour at $37^{\circ} \mathrm{C}$, (ii) overnight at $4{ }^{\circ} \mathrm{C}$.

Figure 3: Mean levels of urinary 8-oxodG determined by ELISA $\left(37{ }^{\circ} \mathrm{C}\right.$ and $\left.4{ }^{\circ} \mathrm{C}\right)$ and liquid chromatography and tandem mass spectrometry (LC-MS/MS).

Figure 4A: Correlation between urinary 8-oxodG concentrations determined by ELISA at $4{ }^{\circ} \mathrm{C}$ and $37{ }^{\circ} \mathrm{C}$. The line of best-fit is shown derived from linear regression analysis, along with Spearman's correlation coefficient $\left(\mathrm{r}_{\mathrm{s}}\right)$ and significance of correlation.

Figure 4B: Correlation between urinary 8-oxodG concentrations determined by ELISA $\left(4{ }^{\circ} \mathrm{C}\right)$ and LC-MS/MS. The line of best-fit is shown derived from linear regression analysis, along with Spearman's correlation coefficient $\left(\mathrm{r}_{\mathrm{s}}\right)$ and significance of correlation.

Figure 5: Competitive ELISA analysis of a single urine sample spiked with varying concentrations of 8-oxoGuo, 8-chloroGuo, 8-oxodGMP, 8-oxodG $\left(20-2.56 \times 10^{-4}\right.$ 
$\mu \mathrm{M})$ or a single-stranded oligodeoxyribonucleotide containing a single 8-oxodG (9.4 $\left.-1.2 \times 10^{-7} \mathrm{nM}\right)$. Incubation of the primary antibody was performed at (A) $37^{\circ} \mathrm{C}$ for one hour, or (B) overnight at $4{ }^{\circ} \mathrm{C}$.

Figure 6: Typical LC-MS/MS SRM ion chromatograms for 8-oxodA (transition $\mathrm{m} / \mathrm{z}$ 268 to 152 ) and the stable isotope internal standard $\left[{ }^{15} \mathrm{~N}_{5}\right] 8-\operatorname{oxodA}$ (transition $\mathrm{m} / \mathrm{z} 273$ to 157) obtained from the analysis of healthy individual's urine following SPE purification. Peak intensities are shown along with an enlarged view of the chromatogram between 28.5 and 45 minutes, encompassing the elution window for any endogenous 8-oxodA (transition $\mathrm{m} / \mathrm{z} 268$ to 152 ). 
Evans - Figure 1

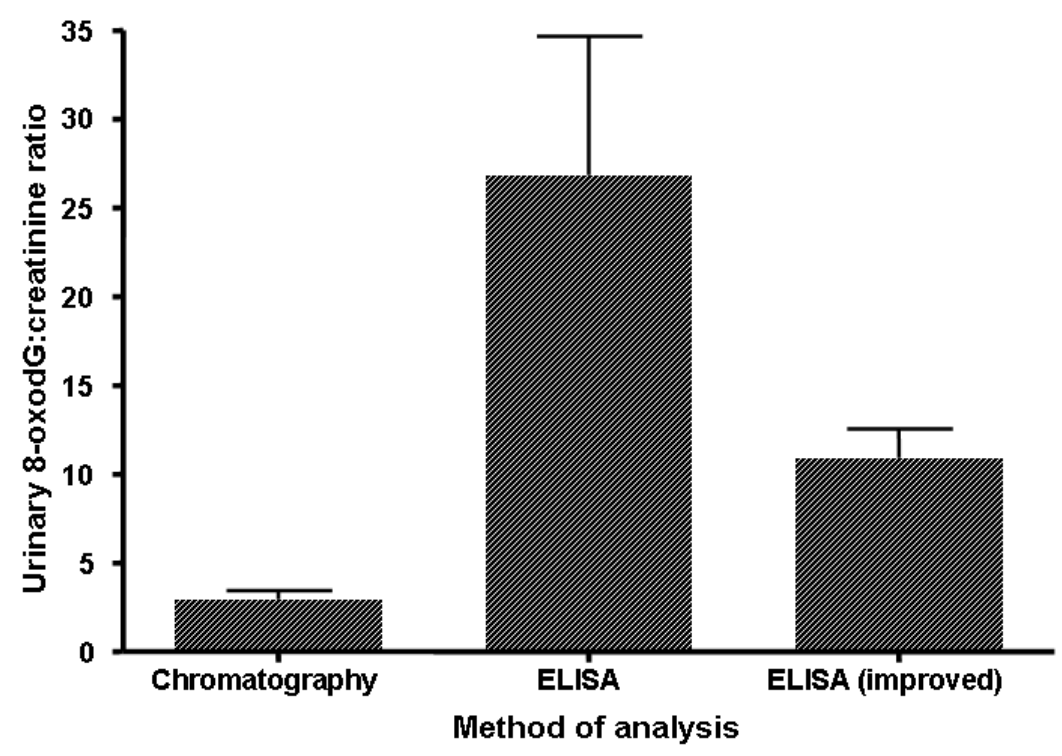


Evans - Figure 2

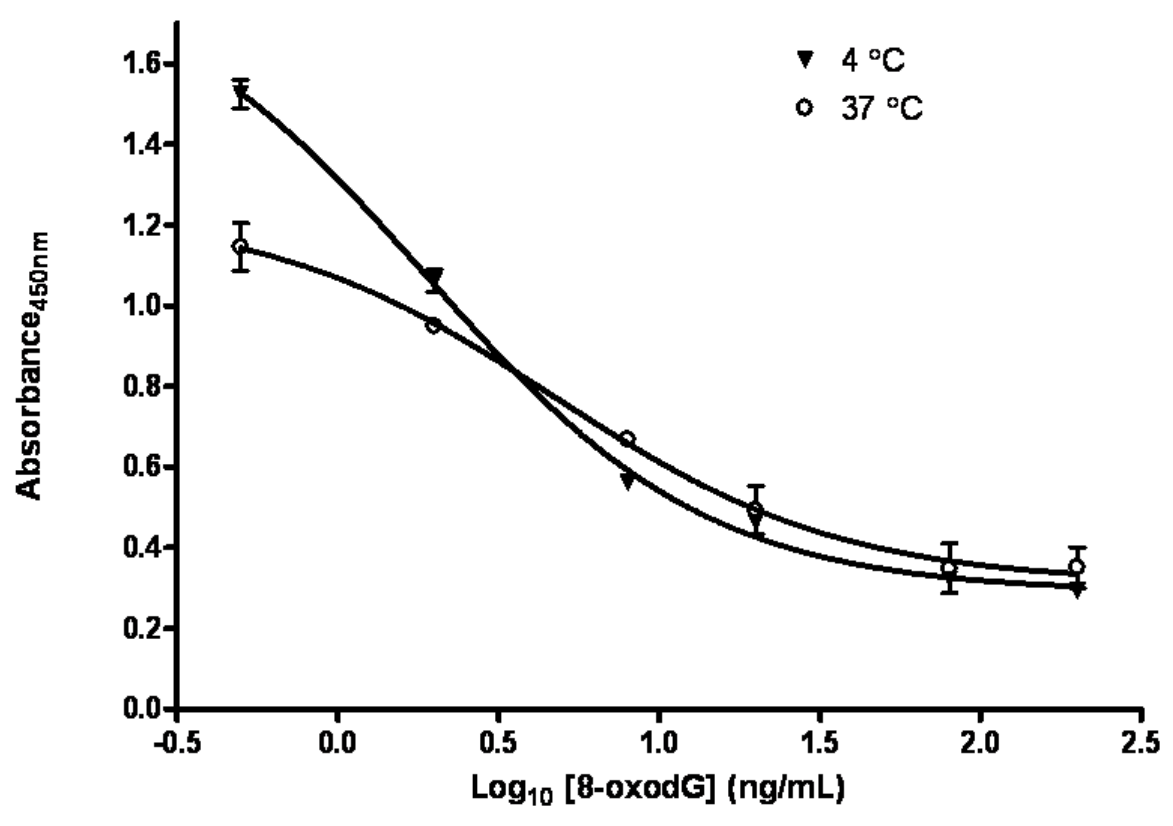


Evans - Fig. 3

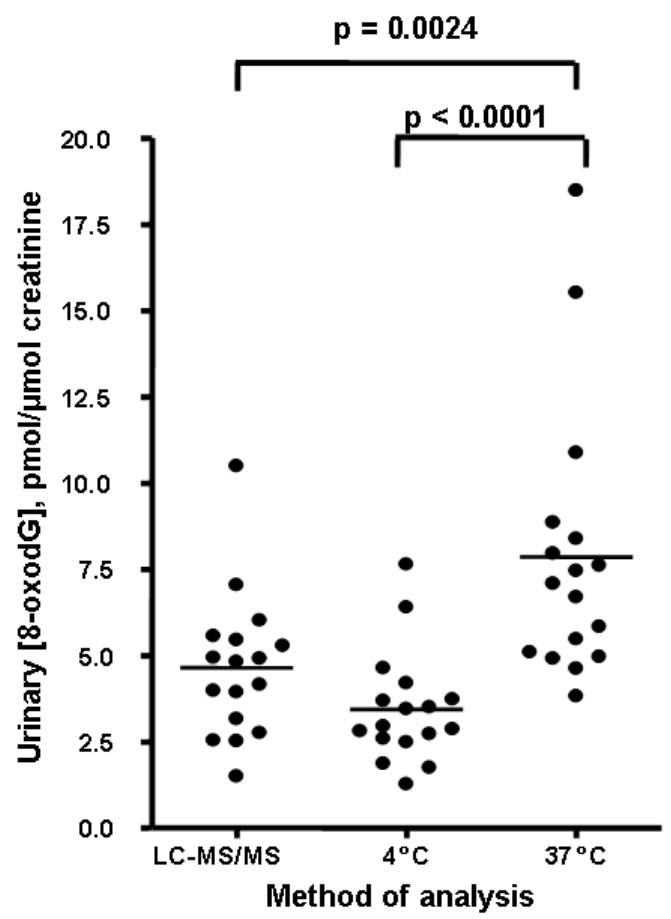


Evans - Fig. 4A

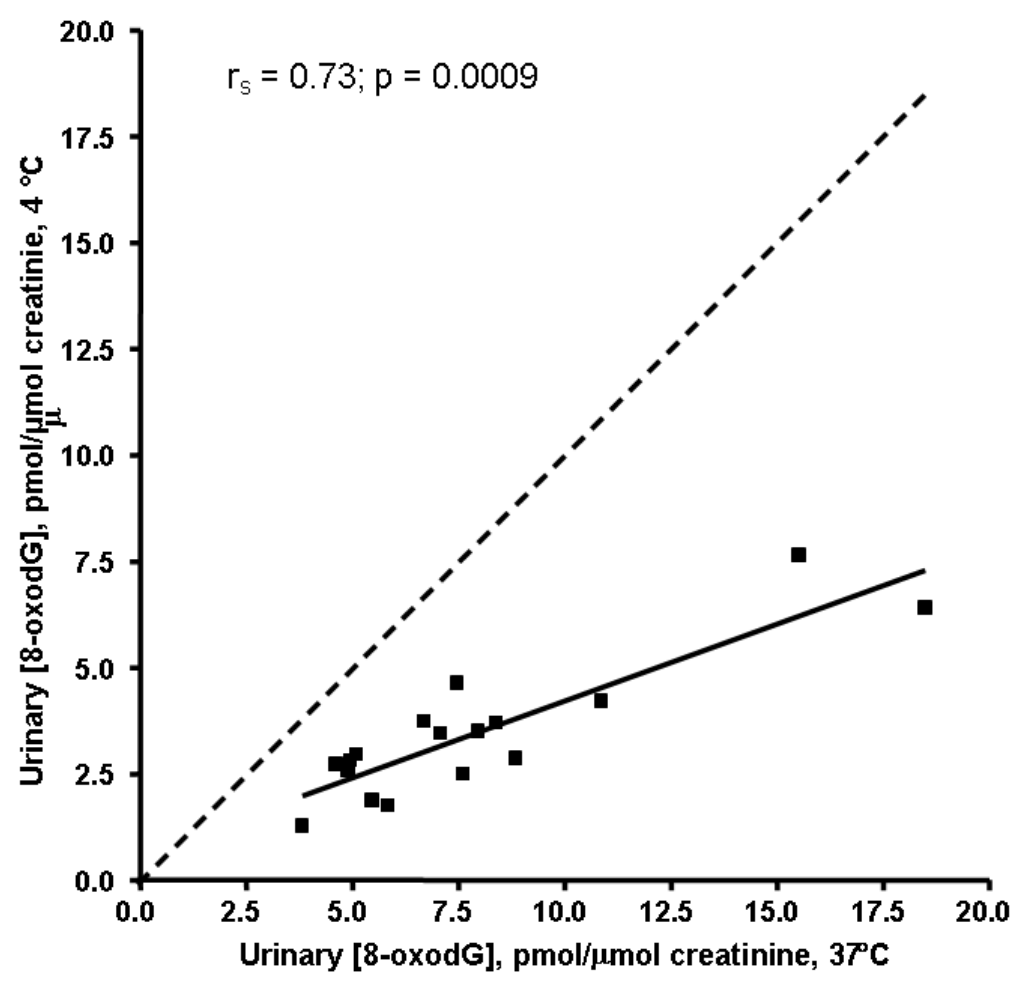

Evans - Fig. 4B

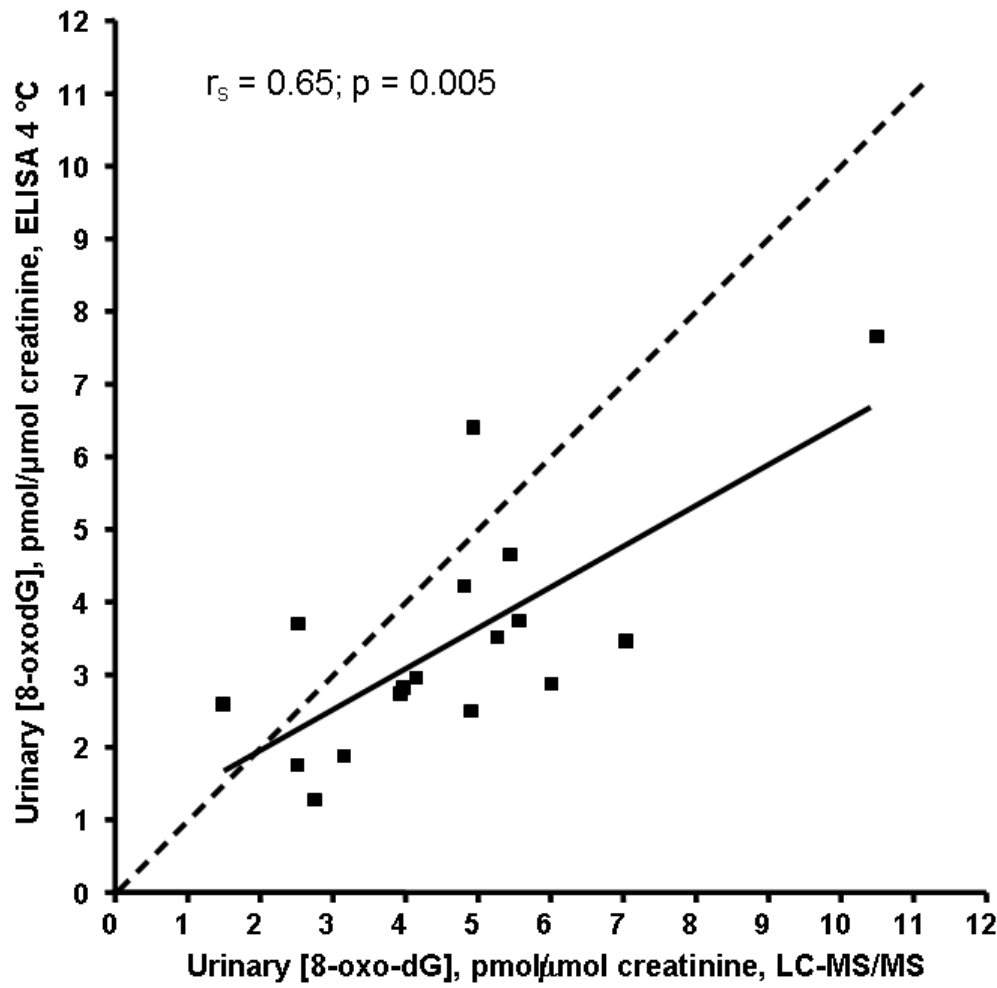


$5 A$

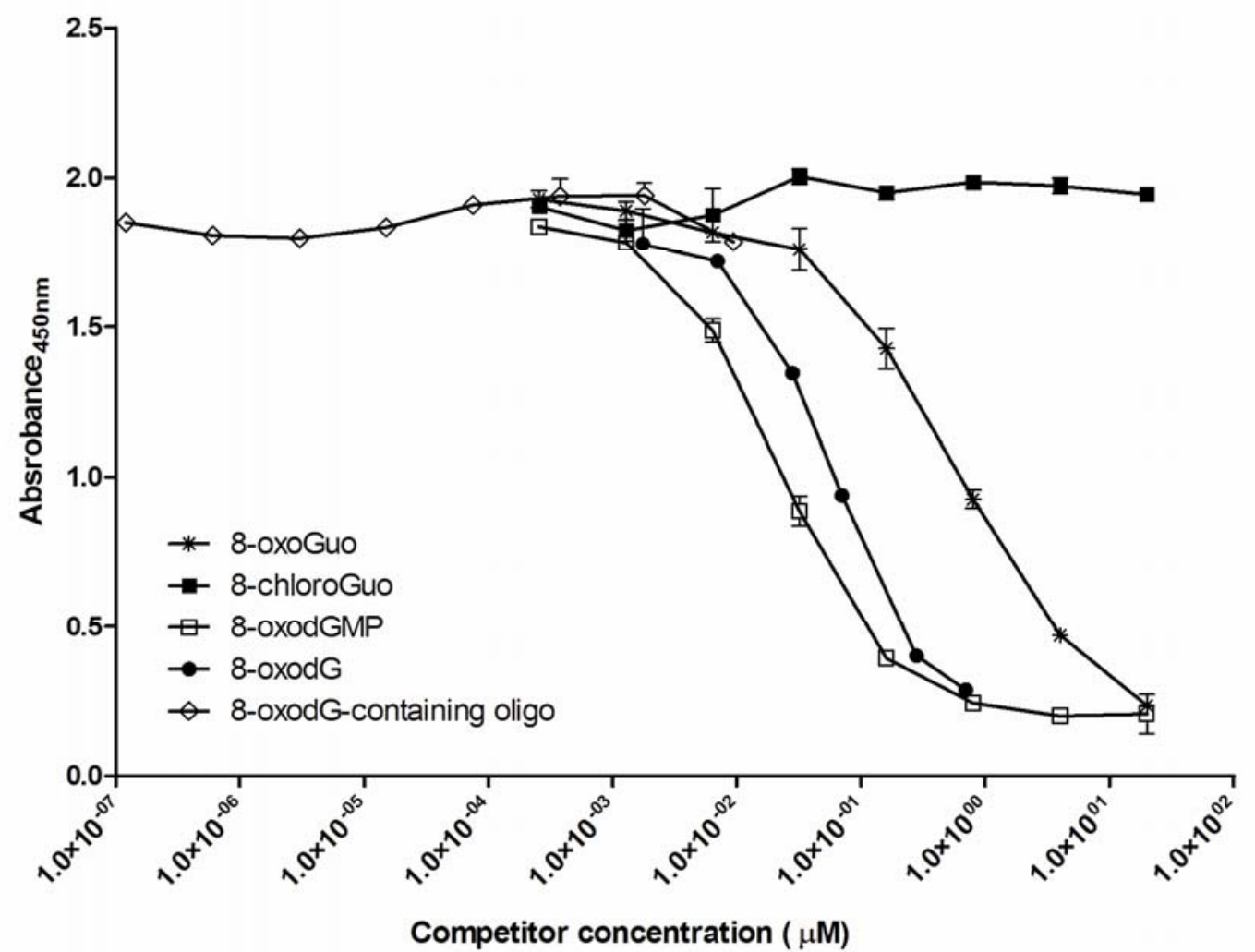

5B

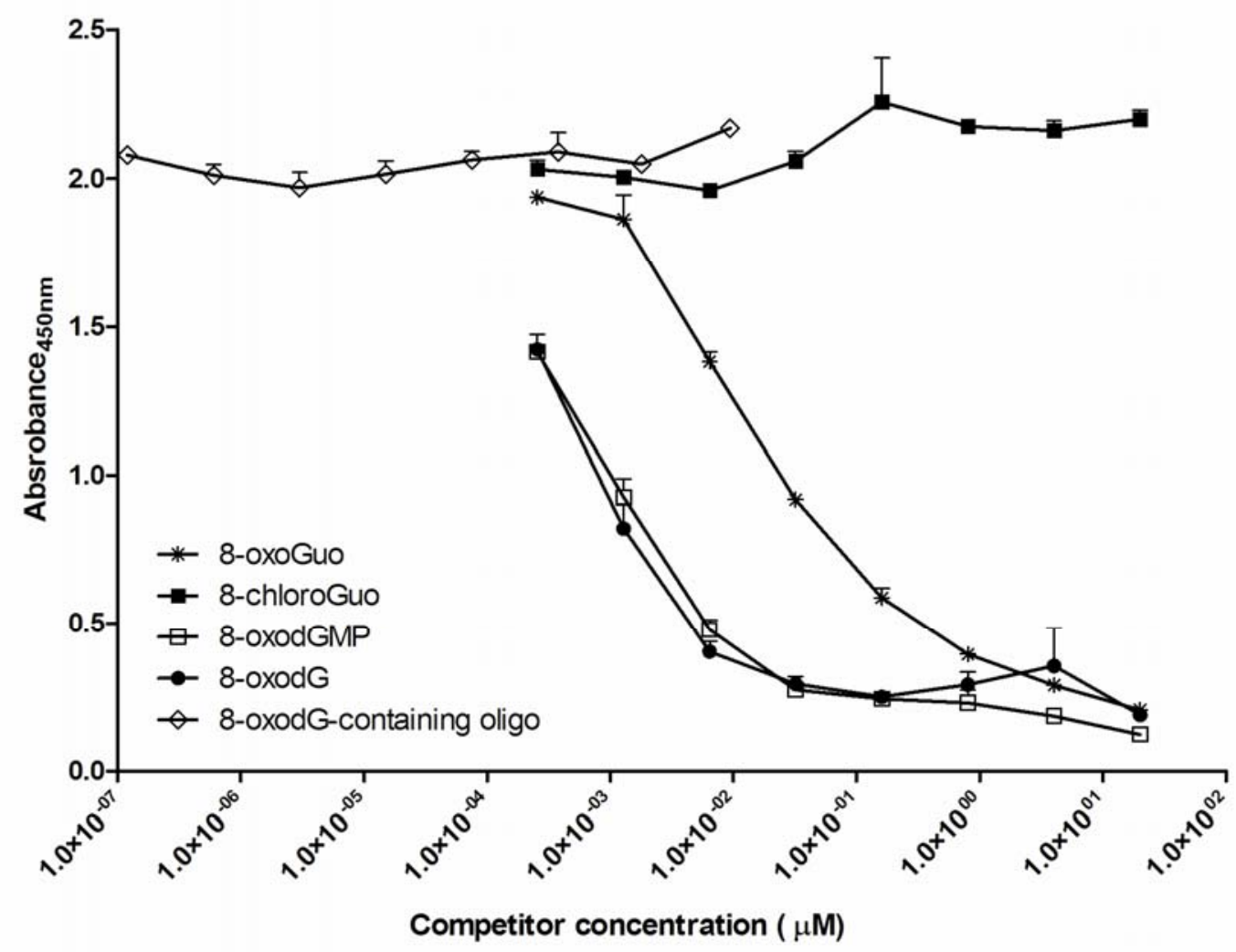


Analysis of urinary 8-oxopurines 30

Evans - Figure 6
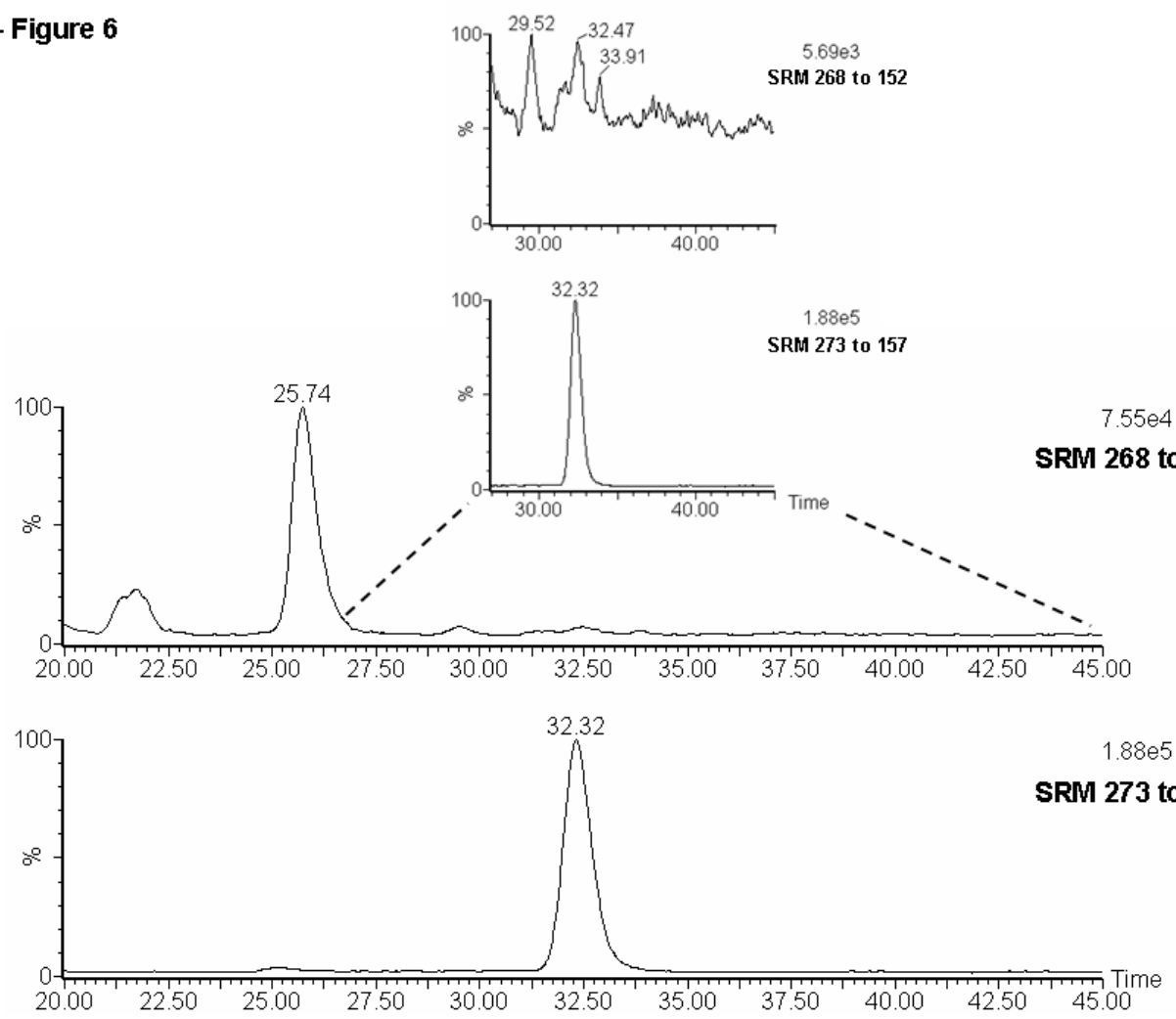